\title{
Host-Microbiome Interactions in Health and Disease
}

Liam O'Mahony, Ph.D.

\section{The Basics of Mucosal Immunology}

The intestinal mucosal immune system must maintain intestinal integrity in the presence of an enormous quantity of external or foreign antigens, such as food proteins and the microbiome. Highly sophisticated cellular and molecular networks need to be constantly coordinated in order to tolerate the presence of such antigens, whereas protective immune responses to potential pathogens must be maintained and induced on demand. The ability to tolerate a wide range of exogenous antigens is a unique feature of the mucosal system that is not seen with the systemic immune system. Tolerance to food and microbial antigens at mucosal surfaces is not a passive process. For example, specialized epithelial cells, M cells, actively transport antigen to underlying lymphoid follicles for immunological processing whereas dendritic cells extend dendrites between epithelial cells in order to sample adherent bacterial species. Upon activation, dendritic cells undergo maturation into potent $\mathrm{T}$ cell stimulatory effector or regulatory dendritic cells and migrate toward the $\mathrm{T}$ cell areas of draining lymphoid organs. Inappropriate immune responses to nonpathogenic antigens significantly contribute to several intestinal pathologies including inflammatory bowel disease and food allergies.

The mucosal immune system is classified as organized or diffuse gut-associated lymphoid tissues (GALT). The organized GALT includes Peyer's patches, mesenteric lymph nodes, and solitary lymphoid follicles in the gut wall where antigen uptake, processing and presentation are facilitated. In contrast, diffuse GALT is a nonorganized system whereby individual cells, such as intra-epithelial lymphocytes (IELs), are dispersed throughout nonlymphoid tissue. Finally, epithelial cells themselves provide a barrier to antigen translocation and actively participate as sensors of luminal contents via the expression of Toll-like receptors (TLRs).

\section{Mechanisms for Microbiome and Host Interactions}

The balance between immune tolerance and inflammation is regulated through the crosstalk between epithelial and immune cells with the intestinal microbiota, involving many signaling pathways and molecules. Direct contact with bacterial-associated structures can activate receptors (eg, TLRs) on host cells, which induce signaling cascades, resulting in both innate and adaptive polarized immune responses (Fig. 1). Following activation, dendritic cells release a variety of molecules, eg, cytokines and metabolites, which determines the polarization of naive $\mathrm{T}$ helper (TH) cells into $\mathrm{TH} 1, \mathrm{TH} 2, \mathrm{TH} 9, \mathrm{TH} 17$, or $\mathrm{T}$ regulatory cells (TREGs). ${ }^{1}$ Importantly, expression of $\mathrm{TH}$ cell-polarizing molecules by mature dendritic cells strongly depends on the conditions during their initial activation as sentinel dendritic cells. These findings imply that the microbiome and dietary components may promote the development of distinct dendritic cell phenotypes by provoking tissues to release mediators involved in polarization (Fig. 2). For example, MHC II-dependent presentation of segmented filamentous bacteria antigens by intestinal dendritic cells promotes the local induction of TH17 lymphocytes. ${ }^{2}$ In contrast, dendritic cells exposed to Bifidobacterium infantis 35624 promote polarization of TREGs and administration of this bacterium to patients with psoriasis, chronic fatigue syndrome, or ulcerative colitis consistently reduced serum levels of proinflammatory biomarkers such as C-reactive protein, possibly mediated by increased numbers of TREG cells. $^{3,4}$

In addition to direct contact with microbial structures, the intestinal microbiome is metabolically active, and microbial metabolites have been shown to exert significant effects on host immune signaling networks. The production of short-chain fatty acids (SCFAs) occurs in the colon

Abbreviations: FMT, fecal microbiota transplantation; GALT, gut-associated lymphoid tissues; GPCR, g-protein coupled receptors; IEL, intra-epithelial lymphocytes; MHC, major histocompatability complex; SCFA, short-chain fatty acid; TLR, Toll-like receptor; TREG, T regulatory cell.

From the Swiss Institute of Allergy and Asthma Research (SIAF), University of Zurich, Davos, Switzerland

Potential conflict of interest: Nothing to report.

View this article online at wileyonlinelibrary.com

(C) 2015 by the American Association for the Study of Liver Diseases

doi: $10.1002 /$ cld.484 


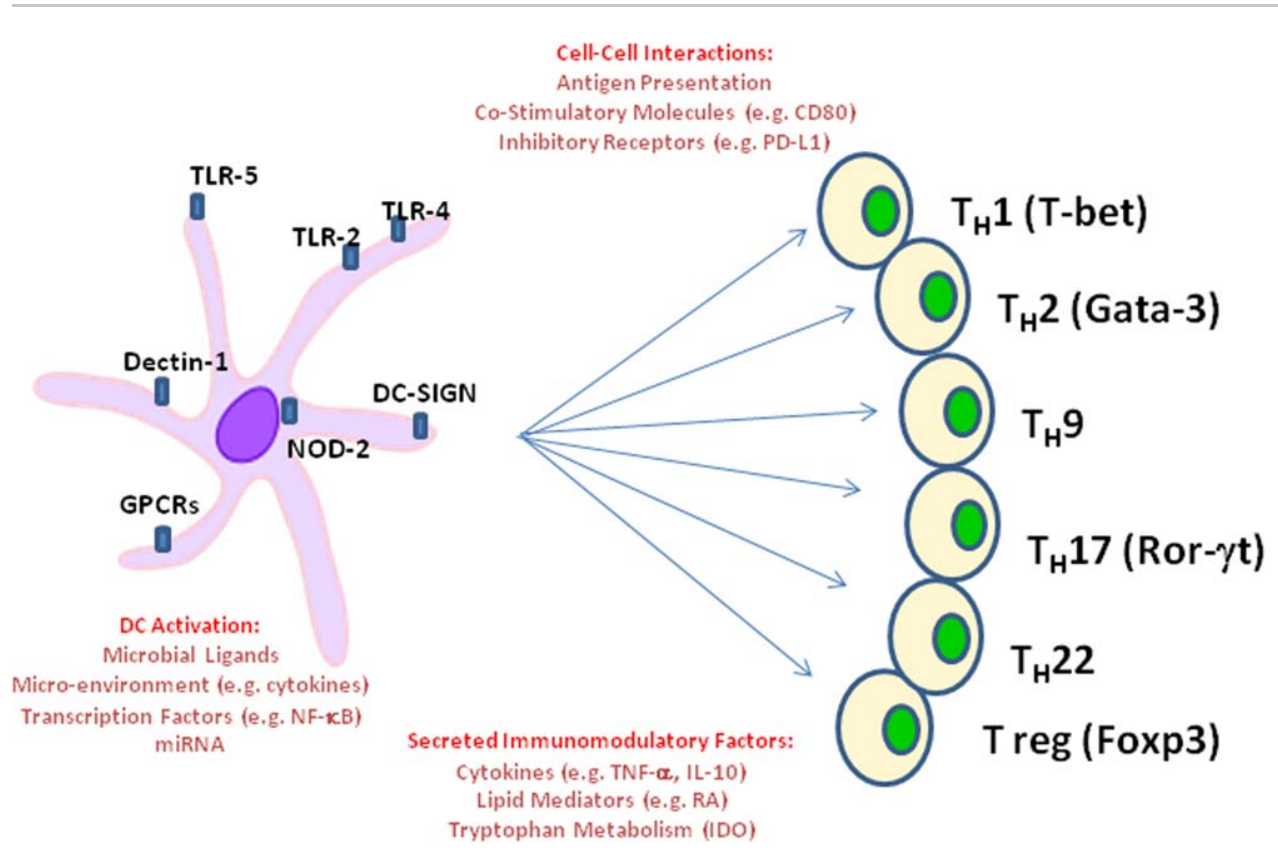

Figure 1 Dendritic cell activation by microbes polarize the adaptive immune response. Dendritic cells recognize microbial components and metabolites via pattern recognition receptors (eg, TLRs, NODs) and GPCRs (eg, histamine receptor 2). Following activation, dendritic cells present antigen, alter cell surface expression of costimulatory or inhibitory molecules, and release mediators such as cytokines or metabolites, which shape the subsequent adaptive immune response.

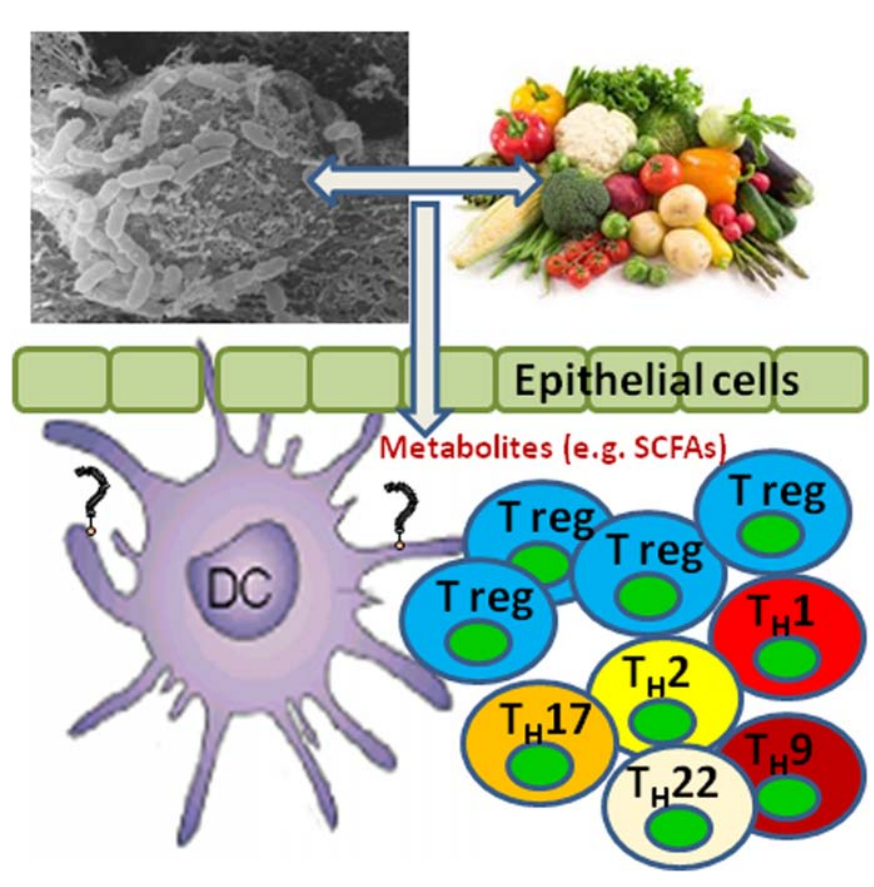

Figure 2 Microbes and metabolites influence mucosal immune regulation. The type of microbes interacting with mucosal cells will influence host immune cell activity. Microbiome metabolism of dietary factors (eg, fiber) will also influence mucosal host cell activity, suggesting that microbiome-dietary interactions are important. following microbiome fermentation of dietary fibers. ${ }^{5}$ Among the SCFAs, butyrate seems to be more potent than acetate or propionate in inducing immunomodulatory effects. Butyrate promotes dendritic cell regulatory activity resulting in the induction of TREG cells and IL10-secreting $T$ cells. These effects were mediated by the $G$ protein-coupled receptors (GPCRs). In addition to SCFAs, the microbiome secretes a wide range of other biologically important metabolites. For example, histamine is secreted by gut microbes, and mucosal histamine levels are increased in patients with irritable bowel syndrome and inflammatory bowel disease. ${ }^{6}$ Histamine modifies chemokine and cytokine secretion by dendritic cells via the GPCR histamine receptor 2 , whereas microbes secreting histamine exert immunoregulatory effects in vivo. ${ }^{7}$

\section{Implications of Disturbed Host-Microbe Interactions for Disease States}

Abnormalities in microbiome composition and/or metabolic activity have been shown in a wide range of disease states, including type-2 diabetes, obesity, inflammatory bowel disease, colorectal cancer, and allergies. ${ }^{8}$ Of note, an increased proportion of gram-negative species with a decrease in autochthonous familiae have been described in patients with cirrhosis, which may contribute to an increased risk of bacterial translocation, systemic infection, and systemic inflammation. ${ }^{9}$ However, in many disease states it 
remains to be determined if microbiome abnormalities precede disease or are the consequence of disease processes.

Efforts to use microbiome-associated therapeutics (eg, probiotics) have clearly shown beneficial effects in animal models, with inconsistent findings in humans probably due to differences in the bacterial strains used. Fecal microbiota transplantation (FMT) is a more radical approach, whereby the entire microbiome from a healthy individual is transplanted into a patient. FMT has repeatedly been shown to be effective in patients infected with Clostridium difficile, with intriguing results in patients with metabolic syndrome. ${ }^{10}$ Further studies are still required to

\section{References}

1. Akdis M, Burgler S, Crameri R, Eiwegger T, Fujita H, Gomez E, et al. Interleukins, from 1 to 37 , and interferon- $\gamma$ : receptors, functions, and roles in diseases. J Allergy Clin Immunol 2011;127:701-721.

2. Goto Y, Panea C, Nakato G, Cebula A, Lee C, Diez MG, et al. Segmented filamentous bacteria antigens presented by intestinal dendritic cells drive mucosal Th17 cell differentiation. Immunity 2014;40:594607.

3. Konieczna P, Groeger D, Ziegler M, Frei R, Ferstl R, Shanahan F, et al. Bifidobacterium infantis 35624 administration induces Foxp3 T regulatory cells in human peripheral blood: potential role for myeloid and plasmacytoid dendritic cells. Gut 2012;61:354-366.

4. Groeger D, O'Mahony L, Murphy EF, Bourke JF, Dinan TG, Kiely B, et al. Bifidobacterium infantis 35624 modulates host inflammatory processes beyond the gut. Gut Microbes 2013;4:325-339. establish the specific benefits of microbiome therapeutics such as probiotics or FMT, but it is clear that hostmicrobiome interactions impact many immune and metabolic processes, and a better description of these interactions at a molecular level will significantly progress this exciting field. In addition, the interaction between dietary factors and the microbiome should be considered in future studies.

\section{CORRESPONDENCE}

Liam O'Mahony, Swiss Institute of Allergy and Asthma Research (SIAF), University of Zurich, Obere strasse 22, 7270 Davos Platz, Switzerland. E-mail: liam.omahony@siaf.uzh.ch

5. Tan J, McKenzie C, Potamitis M, Thorburn AN, Mackay CR, Macia L. The role of short-chain fatty acids in health and disease. Adv Immunol 2014;12 1:91-119.

6. Smolinska S, Jutel M, Crameri R, O'Mahony L. Histamine and gut mucosal immune regulation. Allergy 2014;69:273-281.

7. Frei R, Ferstl R, Konieczna P, Ziegler M, Simon T, Rugeles TM, et al. Histamine receptor 2 modifies dendritic cell responses to microbial ligands. J Allergy Clin Immunol 2013;132:194-204.

8. Frei R, Lauener RP, Crameri R, O'Mahony L. Microbiota and dietary interactions: an update to the hygiene hypothesis? Allergy 2012;67:451-461.

9. Giannelli V, Di Gregorio V, Iebba V, Giusto M, Schippa S, Merli M, Thalheimer $\mathrm{U}$. Microbiota and the gut-liver axis: Bacterial translocation, inflammation and infection in cirrhosis. World J Gastroenterol 2014;20:16795-16810.

10. Xu MQ, Cao HL, Wang WQ, Wang S, Cao XC, Yan F, et al. Fecal microbiota transplantation broadening its application beyond intestinal disorders. World J Gastroenterol. 2015;21:102-111. 